\title{
Computer in der Musik
}




\title{
Helmut Schaffrath (Hrsg.)
}

\section{Computer in der Musik}

Über den Einsatz in Wissenschaft Komposition und Pädagogik

\author{
Mit Beiträgen von \\ Ulrich Blomann \\ Bernd Enders \\ Marc Leman \\ Christoph Micklisch \\ Dirk Reith \\ Helmut Schaffrath \\ Walter Schröder-Limmer \\ Udo Will \\ Ioannis Zannos
}

J. B. Metzlersche Verlagsbuchhandlung

Stuttgart 
CIP-Kurztitelaufnahme der Deutschen Bibliothek

Computer in der Musik:

über den Einsatz in Wissenschaft, Komposition und Pädagogik/

Helmut Schaffrath (Hrsg.).

Mit Beitr. von Ulrich Blomann ... - Stuttgart: Metzler, 1991

ISBN 978-3-476-30330-1

NE: Schaffrath, Helmut [Hrsg.]; Blomann, Ulrich

ISBN 978-3-476-30330-1

ISBN 978-3-476-98901-7 (eBook)

DOI 10.1007/978-3-476-98901-7

Alle Rechte vorbehalten

(C) 1991 Springer-Verlag GmbH Deutschland

Ursprünglich erschienen bei J. B. Metzlersche Verlagsbuchhandlung und Carl Ernst Poeschel Verlag GmbH in Stuttgart 1991

Dieses Werk einschließlich aller seiner Teile ist urheberrechtlich geschützt.

Jede Verwertung außerhalb der engen Grenzen des Urheberrechtsgesetzes

ist ohne Zustimmung des Verlags unzulässig und strafbar.

Das gilt insbesondere für Vervielfältigungen, Übersetzungen,

Mikroverfilmungen und die Einspeicherung und Verarbeitung

in elektronischen Systemen.

Layout: Computersatz Castera, Denkendorf

Umschlagentwurf: Freitag und Häussermann, Gestaltungsagentur, Schwäbisch Gmünd

Druck: Gulde-Druck, Tübingen 


\section{Inhaltsverzeichnis}

Vorwort 6

Helmut Schaffrath

Zum Einsatz von Computern

in Musikwissenschaft und -pädagogik 8

Marc Leman

Künstliche Neuronale Netzwerke

Neue Ansätze zur ganzheitlichen Informationsverarbeitung

in der Musikforschung 27

\section{Ioannis Zannos}

Der Rechner in der Notationsforschung

und musikalischen Analyse - Ein Beispiel 45

\section{Udo Will}

Sampling und Musik-Analyse $\quad 51$

\section{Dirk Reith}

Algorithmische Komposition,

Dargestellt an drei Projekten aus dem elektronischen Studio

der Folkwang-Hochschule Essen 66

\section{Walter Schröder-Limmer}

Kreativer Umgang mit dem Computer

Algorithmische und aleatorische Übungen in Basic 80

\section{Bernd Enders}

Lehr- und Lernprogramme in der Musik 105

\section{Christoph Micklisch}

Heute würde ich alles anders machen

Aufbau, Verwendung und Probleme

einer privaten MIDI-Anlage 131

\section{Ulrich Bloman}

MIDI macht's möglich

Plädoyer für Popularmusikproduktion und -rezeption

an Musikschulen. Ein Erfahrungsbericht 144

Glossar $\quad 152$

Literatur 156 


\section{Vorwort}

Die Idee zu diesem Band entstand aus einer Vorlesungsreihe im Fach Musik der Universität-Gesamthochschule Essen.

Seit geraumer Zeit hatte das Wort >Computer< (am ehesten unter den Studenten) den Schrecken abzulehnender Technik verloren. Die Maschine läßt sich schließlich zum Schreiben von Manuskripten nutzbringend einsetzen. Wer aber im Bereich der Popularmusik ohnehin schon Umgang mit Synthesizern, Drum-Machine oder gar Samplern pflegte, dem konnte der Nutzen eines Computers erst Recht nicht verborgen geblieben sein. Gebrauchsanweisungen zum Umgang mit Software wiederum und kleinere Programmiervorhaben verstellen - so dringend sie gebraucht werden - den Blick für weitereichende Entwicklungen. Die NutzerInnen werden hierfür leider inzwischen besser durch die zahlreichen MIDIClubs informiert als dort, wo Musik beruflich betrieben wird.

Die Aufgabe des Seminars bestand also darin, über die Ränder solcher Teller hinauszuweisen, d.h. möglichst viele Anwendungsbereiche, Ziele und Perspektiven zu vermitteln. Dazu gehören z.B. die teilweise unvereinbaren Hardware-Welten verschiedener Hersteller ebenso wie die unterschiedlichen Softwareprodukte, die Anwendung im Kompositionsbereich, Entwicklungen für die Musikwissenschaften, Perspektiven für den schulischen Musikunterricht und aktuellste Forschungstendenzen. Anders ausgedrückt hätten die Fragen auch lauten können: Was leistet derzeit ein Computer als Musikinstrument, als Kompositionshilfe, als Analyseinstrument, als Lehr- und Lerngerät oder als Notendruckmaschine?

Auch in der Lehrerausbildung sollte man sich den Blick nicht von vordergründig pädagogischem Nutzen trüben lassen. Es galt z.B. zu fragen, was künstliche Intelligenz als letzter Trend für Musiker allgemein zu leisten vermag, und damit mußte der Blick auch auf neuere Entwicklungen in den Wissenschaften fallen. Die vorliegenden Beiträge gehen also von diesen Wissenschaftsbereichen aus, um sich dem pädagogischen über den künstlerischen Bereich zu nähern.

Nicht alle Fragen konnten ausführlich behandelt werden, schon gar nicht in Personalunion. Also galt die Suche denen, die sich auf bestimmten Gebieten spezialisiert hatten: Doktoranden, Komponisten, Pädagogen. Ihnen, den Autoren dieses Bandes und Vortragenden im Seminar, sei ausdrücklich noch einmal gedankt.

Wer sich dem Medium Buch anpassen muß, gerät jedoch in Schwierigkeiten. Demonstrationen von Arbeitsweisen oder gar Klangbeispielen sind unmöglich. Die schlichte Vorführung von Programmen überzeugt im übrigen mehr als das gedruckte Wort, und wer nur praktische Anwendungen beschreibt, der klingt vollends trivial. Wir hoffen trotzdem, mit diesem Kompendium Informationen und z.T. auch etwas Unterhaltung aus dem Seminar in die Buchform gießen zu können.

Noch eine Anmerkung: Es ist in diesem Band von Nutzern die Rede, von Lesern, Komponisten, Teilnehmern, Interessenten usf. Wir haben diese Form nur deshalb belassen oder inkonsequent verändert, weil jede Geschlechtsergänzung zumindest das flüssige Le- 
sen von Texten behindert. Den Leserinnen sei aber versichert: Wir meinen ebenso Nutzerinnen, Komponistinnen, Teilnehmerinnen und Interessentinnen!

Essen, 1991

Der Herausgeber 